\section{Cyberbullying and adolescent mental health: systematic review}

\author{
Cyberbullying e saúde mental dos adolescentes: \\ revisão sistemática
}

\author{
Acoso cibernético y la salud mental de los \\ adolescentes: revisión sistemática
}

\begin{abstract}
Cyberbullying is a new form of violence that is expressed through electronic media and has given rise to concern for parents, educators and researchers. In this paper, an association between cyberbullying and adolescent mental health will be assessed through a systematic review of two databases: PubMed and Virtual Health Library (BVS). The prevalence of cyberbullying ranged from $6.5 \%$ to $35.4 \%$. Previous or current experiences of traditional bullying were associated with victims and perpetrators of cyberbullying. Daily use of three or more hours of Internet, web camera, text messages, posting personal information and harassing others online were associated with cyberbullying. Cybervictims and cyberbullies had more emotional and psychosomatic problems, social difficulties and did not feel safe and cared for in school. Cyberbullying was associated with moderate to severe depressive symptoms, substance use, ideation and suicide attempts. Health professionals should be aware of the violent nature of interactions occurring in the virtual environment and its harm to the mental health of adolescents.
\end{abstract}

Bullying; Adolescent; Mental Health
Sara Mota Borges Bottino 1

Cássio M. C. Bottino 2

Caroline Gomez Regina 1

Aline Villa Lobo Correia 1

Wagner Silva Ribeiro ${ }^{1}$

\section{Resumo}

Cyberbullying, uma nova forma de violência expressa por meio da mídia eletrônica, tem preocupado pais, educadores e pesquisadores. A associação entre cyberbullying e a saúde mental dos adolescentes será revisada. Revisão sistemática em duas bases de dados: PubMed e a Biblioteca Virtual em Saúde (BVS). A prevalência do cyberbullying variou entre 6,5\% a 35,4\%. Bullying tradicional prévio ou atual estava associado às vítimas e agressores do cyberbullying. Uso diário de três ou mais horas de Internet, web câmera, mensagens de texto, postar informações pessoais e assediar outros online estavam associados ao cyberbullying. "Cybervítimas" e cyberbullies tinham mais problemas emocionais, psicossomáticos, dificuldades sociais, e não se sentiam seguros e cuidados na escola. O cyberbullying estava associado à sintomatologia depressiva moderada e grave, uso de substâncias, ideação e tentativas de suicídio. Profissionais de saúde devem conhecer as interações de natureza violenta que ocorrem no ambiente virtual e de seus agravos para a saúde mental dos adolescentes.

Bullying; Adolescente; Saúde Mental 


\section{Introduction}

Access to the Internet and use of text messaging has influenced social interaction among teenagers in recent decades. Most teenagers have a personal computer or mobile phone and communicate daily with several people at the same time, being exposed to the influences of instant contact and its repercussions, which in many cases can be harmful ${ }^{1}$. More recently, cyberbullying, a new form of violence expressed through electronic media, has concerned parents, educators and researchers due to its effects on adolescents' mental health 2 .

The term cyberbullying is derived from traditional bullying behaviors, which are observed mainly among elementary-school students, such as verbal abuse, teasing, insults and threats, as well as physical aggression, such as hitting, kicking, punching and damaging the belongings of others. Such behaviors must occur repeatedly and systematically against an individual who fails or is unable to defend himself or herself, in order to be classified as bullying ${ }^{3}$. Correlates of traditional bullying can be useful to understand youth internet harassment, because this field is much more established. Just over $15 \%$ of children and adolescents are bullied "sometimes" or more frequently with negative health and social challenges consistently reported. Studies revealed that victims report significantly more concurrent health problems, emotional-adjustment problems, school-adjustment problems, and poorer relationships with classmates when compared with non-bullying involved youth 4,5. According to Hawker \& Boulton 6 , being the target of bullying was most strongly related to depression, compared with all other outcomes, in a meta-analysis performed with studies from 23 countries.

Cyberbullying, on the other hand, involves the use of electronic media with the intention of causing harm, humiliation, suffering, fear and despair for the individual who is the target of aggression. These actions can be carried out via email, chat rooms, online voting booths, cell phones and instant messaging 7 . Studies suggest that $20-40 \%$ of teenagers will have at least one cyberbullying experience during their adolescence, and that the number of cybervictims is increasing ${ }^{8}$. Nocentini et al. 9, using a list of behaviors, classified cyberbullying according to the nature of attacks in: (1) written-verbal, which include phone calls, text messages and emails; (2) visual, which involves sending embarrassing images and/or pictures; (3) representation, which refers to more sophisticated attacks, consists of using or stealing someone else's identity to reveal personal information, using his/her account and;
(4) exclusion, which consists of deliberately excluding someone who is a member of the online group.

Cyberbullying may achieve a greater audience than traditional bullying, since it occurs in the virtual space, where free expression is allowed without social control. Additionally, it is difficult to remove information from a website. Dehue et al. 10 suggest three conditions that must be met for a behavior be considered "cyberbullying": the attack must be intentional, occur repeatedly and cause psychological distress. The main damage caused by cyberbullying is to harm the victim's reputation, with repercussions that may be even greater than those observed in traditional bullying. Victimization related to cyberbullying has been associated with social and behavioral problems ${ }^{8}$. In this article we aimed to review and evaluate the associations between cyberbullying and adolescent mental health problems.

\section{Methods}

This review included studies on the prevalence of cyberbullying and its association with mental health problems among adolescents in the general population. Several cyberbullying definitions have been presented in the literature, describing some aggressive, hostile, or harmful act that is perpetrated by a bully through an unspecified type of electronic device. In this review, we used the following definition of cyberbullying, with the aim of unifying the concepts that appear in the literature: "an intentional act of aggression towards another person online”. Mental health problems refer to a recognizable set of emotional problems, symptoms or behaviors associated with considerable distress and substantial interference with personal functions.

\section{Information sources}

We searched for studies published in English, Portuguese and Spanish (languages) in two electronic databases: PubMed and Virtual Health Library (Biblioteca Virtual em Saúde - BVS). The BVS includes data from the Latin American and Caribbean Centre on Health Sciences Information (LILACS), Spanish Bibliographic Index of Health Sciences (SBIHS), MEDLINE, The Cochrane Library and SciELO. The Pan American Health Organization/World Health Organization (PAHO-WHO), together with BIREME (the Latin American and Caribbean Center on Health Sciences Information) provides open access to full-text health related publications from Latin America and the Caribbean. The PRISMA state- 
ment was used to build and elaborate this systematic review and the Effective Public Health Practice Project Quality Assessment Tool (EPHPP) was applied to assess the methodological quality of the articles included 11,12.

\section{Search strategy}

The search strategy was performed on two occasions: in May and June 2013. We searched for the term "cyberbullying" in all fields, with no time limits. The search was conducted using the following keyword combinations: (cyberbullying OR bullying) AND adolescent AND (mental health OR health). Search details were: cyberbullying OR (bullying [Mesh terms] OR bullying [All fields] OR cyberbullying [All fields]) AND (adolescent [MESH terms] OR adolescent [all fields]) AND (Mental Health [MESH terms] OR (mental [All fields] AND Health [All fields]) OR (mental health [All fields]. Article references were screened for additional information.

\section{Study selection}

Articles were evaluated and selected according to the following eligibility criteria: (1) populationbased representative samples of 6 th to 12 th grade students or youth from the ages of 10 to 17; (2) cross-sectional prevalence studies on cyberbullying and; (3) the use of standardized instruments to assess cyberbullying and mental health problems. We excluded studies on specific adolescent populations, such as, adolescents at risk of psychosis, those with intellectual disabilities, developmental issues or obesity, homosexuals or adolescents who were in jail.

Titles and abstracts were assessed independently by two evaluators. Whenever there was disagreement between the evaluators, another reviewer was called to give his opinion.

\section{Data collection}

Data collected: total number of patients, age, sex, study design, sample characteristics, cyberbullying assessment instruments, prevalence and risks factors of cyberbullying, measures and mental health problems related to cyberbullying.

\section{Results}

We found 377 articles in the systematic search and two articles in the manual search. A total of 43 articles were selected. Literature reviews, theoretical essays and qualitative studies were excluded, resulting in 13 articles. Three articles were excluded for the following reasons: (1) the sample was repeated; (2) the study did not inform the prevalence of cyberbullying; and (3) the response rate was below $60 \%$. Figure 1 describes the selection process of the studies included in this review.

Samples were taken from the population of adolescents who were attending the $6^{\text {th }}$ to $12^{\text {th }}$ grades, or aged between 10 and 17 years. Prevalence of cyberbullying varied between $6.8 \%$ to $35.4 \% 13,14,16,17,18,19,20,21,22$. In one study, whose participants were recruited from a popular website, the prevalence was higher: $72 \% 15$. The number of questions used to investigate cyberbullying varied across studies. However, the content of questions was similar and were related to having received or sent messages with the intention of humiliating or causing embarrassment, with threats or disclosure of personal information, photos or photo montage through electronic media, such as internet or mobile phone $14,15,16,17,18,19,20,21,21,22$. We used the follow abbreviation to describe those involved in cyberbullying: cybervictims: Cy-v; cyberbullies (perpetrators of cyberbullying): Cy-b, and those who reported experiences both as a cybervictim and as a cyberbully: Cy-v/Cy-b (Table 1).

Risk factors associated with cyberbullying varied across studies. Nevertheless, in most studies, history of previous or current experiences of traditional bullying was related to cyberbullying $13,14,15,16,17,18,19$. The following factors were associated with cyberbullying: three hours or more of daily internet use 13,16,17, use of instant messaging $15,16,17$, relationship problems $13,16,17$, hyperactivity-inattention problems 17 , behavior problems 17 , school-related problems 14,17,18, and risky behavior on the internet, such as posting personal information, using a web camera and harassment of others online 13,14,15,16 (Table 2).

Between-group comparisons (Cy-v vs. Cy-b vs. Cy-v/Cy-b) showed that a higher proportion of cybervictims came from monoparental families, had psychosomatic problems (headache, abdominal pain and sleep problems), presented higher levels of perceived difficulties, emotional problems, peer and social difficulties, as well as not feeling safe at school and not feeling properly looked after by teachers 17 . The following factors were related to being a cyberbully: headache; high levels of perceived difficulties, not feeling safe in school and not feeling properly looked after by teachers; behavior problems; hyperactivity; smoking, frequent drunkenness; and reduced pro-social behavior (Table 2). Finally, all risk factors above were related to being cyberbullies and cybervictims at the same time 17 .

In a multicenter study that assessed emotional impact of different forms of traditional 


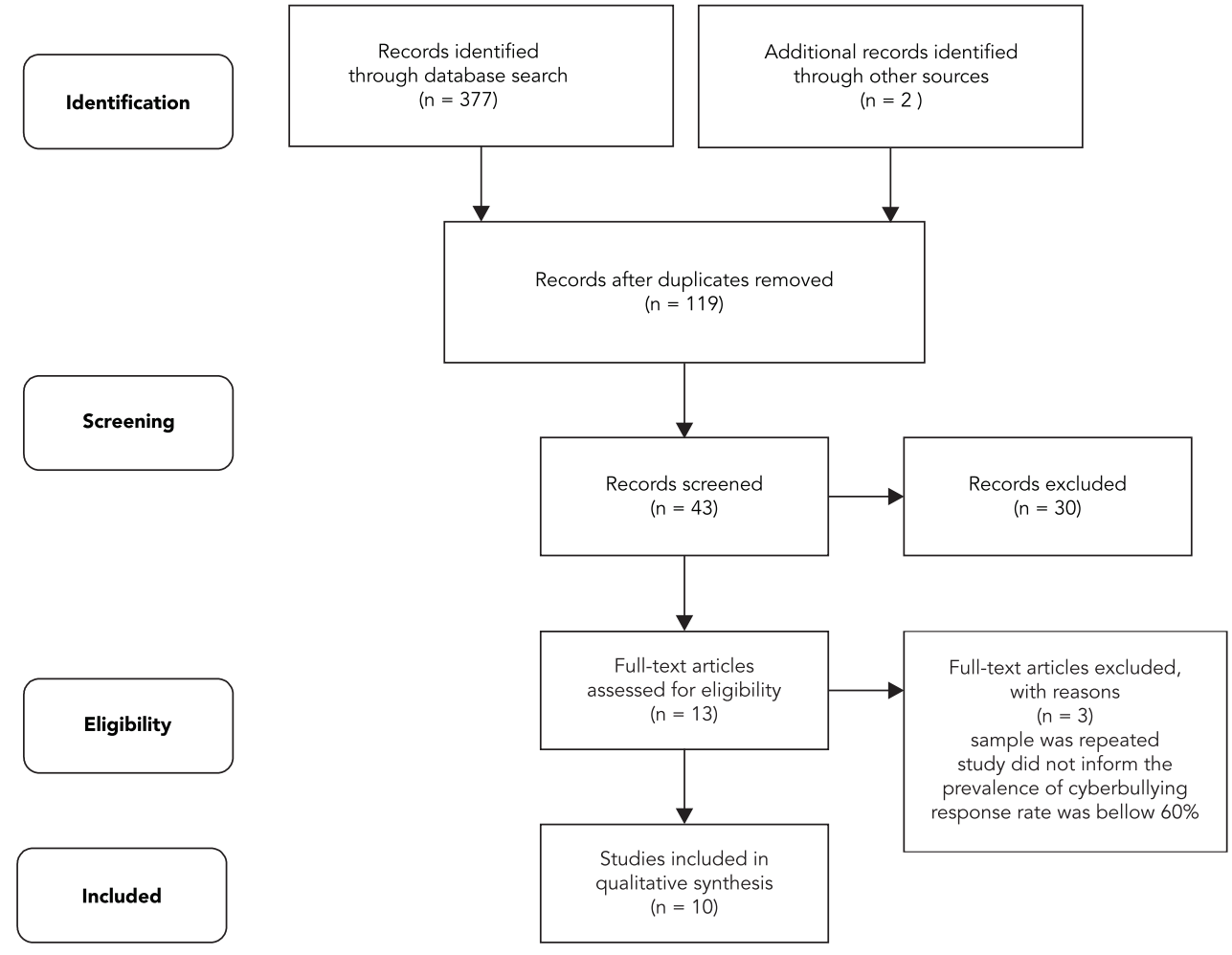

bullying and cyberbullying, $68.5 \%$ of adolescents experienced some negative emotions such as anger, upset, worry, stress, fear and "depressive feelings", while $24.5 \%$ of adolescents did not care about the incidents 22 .

Cyberbullying has been associated with depressive symptomatology in several studies $13,14,18,19$. In three studies $13,14,18$, the likelihood of reporting harassment carried out through the internet was significantly higher among adolescents who presented a more severe depressive symptomatology, as compared to those who presented a mild or absent depressive symptomatology $(\mathrm{OR}=3.38$ 13, OR = 4.38 18) (Table 2).

Adolescents evaluated in the Youth Internet Safety Survey who had depressive symptoms and a history of cyberbullying presented higher scores of emotional distress, as compared to those who did not present depressive symptoms and were not victims of cyberbullying ${ }^{13}$. Five years later, the Second Youth Internet Safety Survey found an increase of $50 \%$ in the prevalence of cyberbullying: $9 \%$ 16. Emotional distress was present in $38 \%$ of the adolescents. The following types of cyberbullying were significantly related to a three-fold increased risk of emotional distress: episodes in which the aggressor was an adult; publication of a picture of the child or adolescent; and episodes accompanied by aggressive contacts off-line, like receiving a telephone call or when the aggressor went to the victim's house 16,17. Preadolescents were significantly more likely to report distress due to the experience of cyberbullying, as compared to adolescents. After adjusting for confounders, the probability of stress and suffering among preadolescents had a four-fold increase $(\mathrm{OR}=3.1$; $\mathrm{p}<0.04) 16$.

Besides depressive symptomatology from moderate to severe intensity $13,14,18,19$, use of substances 20 , suicidal thoughts and suicide attempts were also associated with cyberbullying 18,20,21 (Table 2). Cyberbullying was a predictor of suicide attempts, especially among those who suffer cybervictimization: 1.9 times compared to 1.5 times among the cyberbullying perpetrators 21 . 
Table 1

Characteristics of studies.

\begin{tabular}{|c|c|c|c|c|c|c|c|}
\hline Reference & Country & Study design & $\begin{array}{c}\text { Sample } \\
\text { characteristics }\end{array}$ & Participants & $\begin{array}{l}\text { Participation } \\
\text { rate }\end{array}$ & Cyberbullying measurements & $\begin{array}{l}\text { Quality } \\
\text { of studies }\end{array}$ \\
\hline Ybarra 13 & USA & $\begin{array}{l}\text { Cross-sectional } \\
\text { populational- } \\
\text { based. } \\
\text { Randomly } \\
\text { selected phone } \\
\text { numbers. } \\
\text { Telephone } \\
\text { survey }\end{array}$ & $\begin{array}{c}\text { Regular } \\
\text { Internet urban } \\
\text { users (i.e., at } \\
\text { least six times } \\
\text { in the previous } \\
\text { six months). } \\
\text { One young } \\
\text { person and one } \\
\text { caregiver }\end{array}$ & $\begin{array}{c}\mathrm{N}=1,501 \\
\text { Age: } 10-17 \text { years. } \\
47 \% \text { girls and } \\
53 \% \text { boys. }\end{array}$ & $82 \%$ & $\begin{array}{l}\text { (1) Whether the respondent felt } \\
\text { worried or threatened because } \\
\text { of someone else bothering } \\
\text { or harassing him or her while } \\
\text { online (yes/no) and (2) whether } \\
\text { the respondent felt threatened } \\
\text { or embarrassed because } \\
\text { someone had posted or sent } \\
\text { a message about the young } \\
\text { person for other people to see } \\
\text { (yes/no) }\end{array}$ & Strong \\
\hline Ybarra et al. 16 & USA & $\begin{array}{l}\text { Cross-sectional } \\
\text { populational- } \\
\text { based. } \\
\text { Randomly } \\
\text { selected phone } \\
\text { numbers. } \\
\text { Telephone } \\
\text { survey }\end{array}$ & $\begin{array}{l}\text { Regular } \\
\text { Internet urban } \\
\text { users (i.e., at } \\
\text { least six times } \\
\text { in the previous } \\
\text { six months). } \\
\text { One youth and } \\
\text { one caregiver }\end{array}$ & $\begin{array}{l}\mathrm{N}=1,500 \\
\text { Age: } 10-17 \text { years. } \\
51 \% \text { girls and } \\
49 \% \text { boys }\end{array}$ & $45 \%$ & $\begin{array}{l}\text { (1) In the past year did you } \\
\text { ever feel worried or threatened } \\
\text { because someone was } \\
\text { bothering or harassing you } \\
\text { on-line? } \\
\text { (2) In the past year did anyone } \\
\text { ever use the Internet to } \\
\text { threaten or embarrass you by } \\
\text { posting or sending messages } \\
\text { about you for other people } \\
\text { to see? }\end{array}$ & Strong \\
\hline $\begin{array}{l}\text { Juvonen \& } \\
\text { Gross } 15\end{array}$ & USA & $\begin{array}{l}\text { Cross-sectional. } \\
\text { Anonymous } \\
\text { web-based } \\
\text { survey }\end{array}$ & $\begin{array}{l}\text { Participants } \\
\text { from } 55 \text { states } \\
\text { recruited } \\
\text { through a } \\
\text { popular teen } \\
\text { web site }\end{array}$ & $\begin{array}{l}N=1,454 . \\
\text { Age: } 12-17 \text { years. } \\
75 \% \text { girls and } \\
25 \% \text { boys }\end{array}$ & $\mathrm{NI}$ & $\begin{array}{l}9 \text { questions about aggressive } \\
\text { behavior on the Internet }\end{array}$ & Weak \\
\hline $\begin{array}{l}\text { Hinduja \& } \\
\text { Patchin } 21\end{array}$ & USA & $\begin{array}{l}\text { Cross-sectional, } \\
\text { randomized. } \\
\text { Self-reported } \\
\text { questionnaire }\end{array}$ & $\begin{array}{c}36 \text { middle } \\
\text { schools from } \\
\text { the largest } \\
\text { school district } \\
\text { of USA. } \\
\text { Representative } \\
\text { sample }\end{array}$ & $\begin{array}{l}N=1,963 \\
6^{\text {th-}-8 \text { th }} \text { grades. } \\
48 \% \text { girls and } \\
52 \% \text { boys }\end{array}$ & $96 \%$ & $\begin{array}{c}14 \text { questions: } 9 \text { questions } \\
\text { about cybervictimization and } 5 \\
\text { question about cyberbullying } \\
\text { offending }\end{array}$ & Moderate \\
\hline $\begin{array}{l}\text { Sourander et } \\
\text { al. } 17\end{array}$ & Finland & $\begin{array}{c}\text { Cross-sectional } \\
\text { populational- } \\
\text { based. } \\
\text { Self-reported } \\
\text { questionnaire }\end{array}$ & $\begin{array}{l}\text { Two cities in north } \\
\text { and south of } \\
\text { Finland. } \\
\text { All children } \\
\text { attending the } \\
\text { seventh and ninth } \\
\text { grade, except } \\
\text { for children } \\
\text { attending classes } \\
\text { for the physically or } \\
\text { mentally disabled }\end{array}$ & $\begin{array}{l}\mathrm{N}=2,215 . \\
7^{\text {th }-9 \text { th }} \text { grades. } \\
50 \% \text { girls and } \\
50 \% \text { boys }\end{array}$ & $90.9 \%$ & $\begin{array}{l}\text { Two general questions about } \\
\text { cyberbullying, defined as: } \\
\text { "cyberbullying is when someone } \\
\text { repeatedly makes fun of another } \\
\text { person online or repeatedly } \\
\text { picks on another person through } \\
\text { e-mail or text messages or when } \\
\text { someone posts something online } \\
\text { about another person that they } \\
\text { don't like": } \\
\text { (1) During the past } 6 \text { months, how } \\
\text { often have you been cyberbullied? } \\
\text { (2) During the past } 6 \text { months, how } \\
\text { often have you cyberbullied others }\end{array}$ & Strong \\
\hline
\end{tabular}

(continues) 
Table 1 (continued)

\begin{tabular}{|c|c|c|c|c|c|c|c|}
\hline Reference & Country & Study design & $\begin{array}{c}\text { Sample } \\
\text { characteristics }\end{array}$ & Participants & $\begin{array}{l}\text { Participation } \\
\text { rate }\end{array}$ & Cyberbullying measurements & $\begin{array}{l}\text { Quality } \\
\text { of studies }\end{array}$ \\
\hline Wang et al. 19 & USA & $\begin{array}{l}\text { Cross-sectional } \\
\text { populational- } \\
\text { based. } \\
\text { Self reported } \\
\text { questionnaire }\end{array}$ & $\begin{array}{l}\text { A U.S. } \\
\text { nationally- } \\
\text { representative } \\
\text { sample of } \\
\text { adolescents }\end{array}$ & $\begin{array}{c}\mathrm{N}=7,313 \text {. } \\
\text { 6th-10th grades. } \\
42.3 \% \text { girls and } \\
56.7 \% \text { boys }\end{array}$ & $\mathrm{NI}$ & $\begin{array}{l}\text { Two questions about: (1) having } \\
\text { suffered bullying through } \\
\text { computer: messages or photos; } \\
\text { (2) having suffered bullying } \\
\text { through cellular }\end{array}$ & Strong \\
\hline $\begin{array}{l}\text { Ortega et } \\
\text { al. } 22\end{array}$ & $\begin{array}{l}\text { Italy, } \\
\text { Spain, } \\
\text { England }\end{array}$ & $\begin{array}{l}\text { Cross-sectional, } \\
\text { multicentric. } \\
\text { Self-reported } \\
\text { questionnaire }\end{array}$ & $\begin{array}{l}\text { Urban students. } \\
\text { Representative } \\
\text { sample }\end{array}$ & $\begin{array}{l}\mathrm{N}=5,862 . \\
\text { Age: } 12-16 \text { years. } \\
8 \text { th-, } 10 \text { th and } 12^{\text {th }} \\
\text { grades. } \\
48.8 \% \text { girls and } \\
52.2 \% \text { boys }\end{array}$ & $\mathrm{NI}$ & $\begin{array}{l}12 \text { questions to cellular phone; } \\
12 \text { questions to internet; } \\
\text { DAPHNE questionnaire (Genta } \\
\text { et al. }{ }^{33)}\end{array}$ & Moderate \\
\hline $\begin{array}{l}\text { Schneider et } \\
\text { al. } 18\end{array}$ & USA & $\begin{array}{l}\text { Census: (86\%) } \\
\text { of Boston } \\
\text { metropolitan } \\
\text { area. } \\
\text { Self-reported } \\
\text { questionnaire }\end{array}$ & $\begin{array}{l}22 \text { high school } \\
\text { urban students, } \\
\text { middle and } \\
\text { upper-middle } \\
\text { class families }\end{array}$ & $\begin{array}{l}\mathrm{N}=20,406 \text {. } \\
\text { 9th to } 12 \text { th grades. } \\
50.4 \% \text { girls and } \\
49.6 \% \text { boys }\end{array}$ & $88.1 \%$ & $\begin{array}{l}\text { One question: "How many } \\
\text { times someone used } \\
\text { the internet, cellular or } \\
\text { other means of electronic } \\
\text { communication to intimidate, } \\
\text { provoke or threaten you?" }\end{array}$ & Strong \\
\hline $\begin{array}{l}\text { Litwiller \& } \\
\text { Brausch } 20\end{array}$ & USA & $\begin{array}{l}\text { Cross-sectional, } \\
\text { randomized. } \\
\text { Self-reported } \\
\text { questionnaire }\end{array}$ & $\begin{array}{l}27 \text { high schools } \\
\text { public students, } \\
\text { from seven- } \\
\text { county region. } \\
\text { Representative } \\
\text { sample. } \\
\text { Rural Midwest } \\
\text { region }\end{array}$ & $\begin{array}{c}\mathrm{N}=4,693 \text {. } \\
9 \text { th }-12^{\text {th }} \text { grades. } \\
47 \% \text { girls and } \\
53 \% \text { boys }\end{array}$ & $65 \%$ & $\begin{array}{l}\text { Three questions about } \\
\text { cyberbullying: (1) someone } \\
\text { spread rumors about you } \\
\text { online in chats, website, by } \\
\text { emails or text messages?; } \\
\text { (2) someone posted } \\
\text { compromising or inappropriate } \\
\text { photos of you online?; (3) } \\
\text { someone sent you aggressive } \\
\text { messages or messages with } \\
\text { threats by e-mail or text }\end{array}$ & Strong \\
\hline Chang et al. 14 & Taiwan & $\begin{array}{l}\text { Cross-sectional, } \\
\text { randomized. } \\
\text { Self-reported } \\
\text { questionnaire }\end{array}$ & $\begin{array}{l}26 \text { urban high } \\
\text { schools, two } \\
\text { cities. } \\
\text { Representative } \\
\text { sample }\end{array}$ & $\begin{array}{c}\mathrm{N}=2,992 . \\
10 \text { th } \text { grade. } \\
48 \% \text { girls and } \\
52 \% \text { boys }\end{array}$ & $80 \%$ & $\begin{array}{l}12 \text { questions: six } \\
\text { cybervictimization (how often } \\
\text { has someone: posted rude } \\
\text { comments; embarrassing } \\
\text { photos; spread rumours; } \\
\text { threatening comments; } \\
\text { talked about sex online) and } \\
\text { six cyberbullying (how often } \\
\text { have you ever: posted rude } \\
\text { comments; sent embarrassing } \\
\text { photos; spread rumours; made } \\
\text { threatening comments; talked } \\
\text { about sex online) }\end{array}$ & Strong \\
\hline
\end{tabular}

NI: not informed. 
Table 2

Cyberbullying prevalence and mental health problems.

\begin{tabular}{|c|c|c|c|c|}
\hline Authors & $\begin{array}{l}\text { Prevalence of } \\
\text { cyberbullying }\end{array}$ & Risk factors related to cyberbullying & $\begin{array}{l}\text { Measures of mental health } \\
\text { problems }\end{array}$ & $\begin{array}{c}\text { Mental health problems related } \\
\text { to cyberbullying }\end{array}$ \\
\hline Ybarra 13 & $6.5 \%$ & 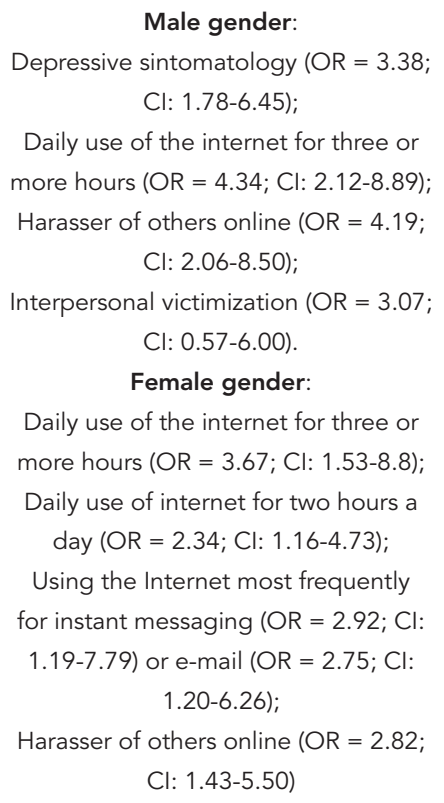 & $\begin{array}{l}\text { Depressive symptoms of DSM- } \\
\text { IV } 34\end{array}$ & $\begin{array}{l}\text { Symptoms of major depression } \\
\qquad(\mathrm{OR}=3.38 ; \mathrm{Cl}: 1.78-6.45)\end{array}$ \\
\hline $\begin{array}{l}\text { Ybarra et } \\
\text { al. } 16\end{array}$ & $9 \%$ & $\begin{array}{l}\text { Harassing others online }(\mathrm{OR}=3.6 ; \mathrm{Cl} \text { : } \\
\qquad 2.3-5.7) ; \\
\text { Using internet for instant messaging, } \\
\text { blogging, and chat room }(\mathrm{OR}=3.4 ; \\
\mathrm{Cl} \text { : } 0.9-6.3) ; \\
\text { Borderline/clinically significant social } \\
\text { problems (OR }=2.4 ; \mathrm{Cl}: 1.2-4.4) \\
\text { Interpersonal victimization }(\mathrm{OR}=1.5 \\
\mathrm{Cl} \text { : } 1.0-2.2)\end{array}$ & $\begin{array}{l}\text { Youth Self-Report of Child } \\
\text { Behavior Checklist } \\
\text { (Achenbach 35) }\end{array}$ & $\begin{array}{l}\text { Emotional distress: } 38 \% \text { of the } \\
\text { harassed youth reported distress } \\
\text { associated; } \\
\text { Characteristics associated with } \\
\text { emotional distress among } \\
\text { targets of harassment incident: } \\
\text { preadolescence (OR }=0.5 .5 ; \mathrm{Cl} \text { : } 1.5 \text { - } \\
\text { 19.3), adult harasser (OR }=4.1 ; \mathrm{Cl} \text {; } \\
\text { 1.4-11.6); } \\
\text { Being asked to send a picture of } \\
\text { oneself (OR }=3.2 ; \mathrm{Cl} \text { : } 1.2-8.4) \text {; } \\
\text { Aggressive offline contact (OR }=3.9 \text {; } \\
\mathrm{Cl}: 1.5-10.1)\end{array}$ \\
\hline $\begin{array}{l}\text { Juvonen \& } \\
\text { Gross } 15\end{array}$ & $72 \%$ & $\begin{array}{c}\text { School bullying }(\mathrm{OR}=6.87 \\
\mathrm{Cl} \text { : } 4.90-9.62) \\
\text { Daily use of the internet for three } \\
\text { hours or more }(\mathrm{OR}=1.45 \\
\mathrm{Cl} \text { : } 1.04-2.02) \\
\text { Instant messaging }(\mathrm{OR}=2.84 \\
\mathrm{Cl} \text { : } 1.08-7.49) \\
\text { Webcam (OR }=1.50 ; \mathrm{Cl}: 1.04-2.14)\end{array}$ & $\begin{array}{l}\text { Social anxiety scale } \\
\text { with } 5 \text { items } \\
\text { (La Greca \& Lopez }{ }^{36} \text { ) }\end{array}$ & $\begin{array}{l}\text { Social anxiety }(\beta=0.31 \\
\qquad p<0.01)\end{array}$ \\
\hline $\begin{array}{l}\text { Hinduja \& } \\
\text { Patchin } 21\end{array}$ & $\begin{array}{c}\text { Cy-v: } 5.7-18.3 \% \\
\text { Cy-b: } 9-23.1 \%\end{array}$ & Not found & $\begin{array}{c}\text { Four items about suicide } \\
\text { attempt and suicide adapted } \\
\text { from American School Health } \\
\text { Associations } 37\end{array}$ & $\begin{array}{c}\text { Suicidal ideation: } \\
\text { Cybervictim }(\mathrm{OR}=1.94 ; \\
\mathrm{p}<0.001) ; \\
\text { Cyberbully }(\mathrm{OR}=1.49 ; \mathrm{p}<0.05)\end{array}$ \\
\hline
\end{tabular}

(continues) 
Table 2 (continued)

\begin{tabular}{|c|c|c|c|c|}
\hline Authors & $\begin{array}{l}\text { Prevalence of } \\
\text { cyberbullying }\end{array}$ & Risk factors related to cyberbullying & $\begin{array}{l}\text { Measures of mental health } \\
\text { problems }\end{array}$ & $\begin{array}{l}\text { Mental health problems } \\
\text { related to cyberbullying }\end{array}$ \\
\hline $\begin{array}{l}\text { Sourander } \\
\text { et al. } 17\end{array}$ & $\begin{array}{l}17.6 \% \\
\text { Cy-v: } 4.8 \% \\
\text { Cy-b: } 7.4 \% \\
\text { Cy-v/b: } 5.4 \%\end{array}$ & $\begin{array}{l}\text { Cybervictimization: not living with } \\
\text { both biological parents; perceived } \\
\text { difficulties; emotional problems and } \\
\text { peer problems; headache, abdominal } \\
\text { pain, sleeping problems, not feeling } \\
\text { safe at school and uncared about by } \\
\text { teachers. } \\
\text { Traditional victimization and school } \\
\text { bullying. } \\
\text { Cyberbullies: high level of perceived } \\
\text { difficulties, hyperactivity, conduct } \\
\text { problems, and lowprosocial behavior. } \\
\text { Traditional bullying. Cybervictim and } \\
\text { cyberbully were associated with all } \\
\text { these risk factors above }\end{array}$ & $\begin{array}{c}\text { Strengths and Difficulties } \\
\text { Questionnaire - SDQ (Goodman } \\
\text { 38) }\end{array}$ & 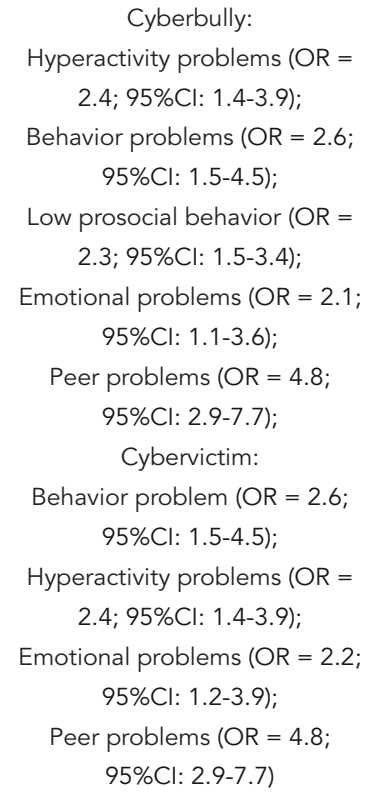 \\
\hline $\begin{array}{l}\text { Wang et } \\
\text { al. } 19\end{array}$ & $10.1 \%$ & $\begin{array}{l}\text { Male-gender. } \\
\text { 6-8 grade }\end{array}$ & $\begin{array}{c}\text { Depression: } \\
\text { Six items: sadness; irritation, bad } \\
\text { mood; hopelessness; changes } \\
\text { in appetite; sleep; concentration } \\
\text { problems in the past } 30 \text { days } \\
\text { (Dahlberg et al. }{ }^{39} \text { ) }\end{array}$ & $\begin{array}{l}\text { Depression, medically } \\
\text { attended injuries, medicine } \\
\text { use for sleeping problems and } \\
\text { nervousness * }\end{array}$ \\
\hline $\begin{array}{l}\text { Ortega et } \\
\text { al. } 22\end{array}$ & $\begin{array}{c}\text { England: } 2.0 \% \\
\text { mobile phone, } 2.6 \% \\
\text { Internet; } \\
\text { Italy: } 2.2 \% \text { mobile } \\
\text { phone; } 1.9 \% \\
\text { Internet; } \\
\text { Spain: } 0.5 \% \text { mobile } \\
\text { phone } 1.3 \% \text { Internet }\end{array}$ & $\mathrm{NI}$ & $\begin{array}{l}\text { DAPHNE questionnaire } \\
\text { (Genta et al. }{ }^{33)}\end{array}$ & $\begin{array}{l}\text { Mobile phone cyberbullying: } \\
\text { angry - 35.8\%; not bothered - } \\
\text { 25.4\%;worried - } 19.5 \% \text {; upset } \\
\text { - 18.3\%; stressed - } 13.3 \% \text {; afraid } \\
\text { - 13.3\%; depressed - } 10.7 \% \\
\text { Internet cyberbullying: not } \\
\text { bothered - 35.7\%; angry - } \\
\text { 33.7\%; upset - } 19.5 \% \text {; worried } \\
\text { - } 15.5 \% \text {; afraid - } 12.8 \% \text {; } \\
\text { depressed - } 10.3 \%\end{array}$ \\
\hline $\begin{array}{l}\text { Schneider et } \\
\text { al. } 18\end{array}$ & $15.8 \%$ & $\begin{array}{l}\text { Female gender; non-white/mixed } \\
\text { race; non heterosexuality identified; } \\
\text { lower school performance; lower } \\
\text { school attachment }\end{array}$ & $\begin{array}{l}\text { Centers for Disease Control and } \\
\text { Prevention 40; } \\
\text { Health and Risk Behaviors of } \\
\text { Massachusetts Youth } 41\end{array}$ & $\begin{array}{c}\text { Distress: } \\
\text { Depressive symptoms (OR= } \\
\text { 4.38; 99\%Cl: 3.76-5.10); } \\
\text { Self-injury (OR = 4.79; 99\% } \\
\text { 4.06-5.65); } \\
\text { Suicidal ideation (OR = 3.35; } \\
\text { 99\%Cl: } 2.71-4.13) ; \\
\text { Suicide attempt (OR = 5.04; } \\
\text { 99\%Cl: 3.88-6.55); } \\
\text { Suicide attempt that required } \\
\text { medical treatment (OR = 5.42; } \\
\text { 99\%Cl: 3.56-8.26) }\end{array}$ \\
\hline
\end{tabular}

(continues) 
Table 2 (continued)

\begin{tabular}{|c|c|c|c|c|}
\hline Authors & $\begin{array}{l}\text { Prevalence of } \\
\text { cyberbullying }\end{array}$ & Risk factors related to cyberbullying & $\begin{array}{c}\text { Measures of mental health } \\
\text { problems }\end{array}$ & $\begin{array}{l}\text { Mental health problems } \\
\text { related to cyberbullying }\end{array}$ \\
\hline $\begin{array}{l}\text { Litwiller \& } \\
\text { Brausch } 20\end{array}$ & $23 \%$ & Not founded & $\begin{array}{c}\text { Suicidal behavior: } \\
\text { Center for Disease and } \\
\text { Control 40; } \\
\text { Substance use: } \\
17 \text { items from Monitoring the } \\
\text { Future Survey (Johnston } 42 \text { ); } \\
\text { Violent behavior: } 4 \text { items from } \\
\text { Center for Disease and Control, } \\
200840 \text {; } \\
\text { Sexual behavior: } 5 \text { items from } \\
\text { Center for Disease and } \\
\text { Control } 40\end{array}$ & $\begin{array}{c}\text { Substance use (estimate =0.33; } \\
\qquad 95 \% \mathrm{Cl} \text { : } 0.28-0.35 \text { ); } \\
\text { Violent behavior (estimate = } \\
0.16 ; 95 \% \mathrm{Cl}: 0.14-0.19) ; \\
\text { Non safe sex behavior } \\
\text { (estimate }=0.005 ; 95 \% \mathrm{Cl}: 0.00- \\
0.15 \text { ); } \\
\text { Suicidal behavior } \mathrm{R}^{2} \text { adj }=0.67\end{array}$ \\
\hline $\begin{array}{l}\text { Chang et } \\
\text { al. } 14\end{array}$ & $\begin{array}{c}35.4 \% \\
\text { Cy-v: } 18.4 \% \\
\text { Cy-b: } 5.4 \% \\
\text { Cy-v/Cy-b: } 11.2 \%\end{array}$ & $\begin{array}{c}\text { Male (OR = 1.57; } 95 \% \mathrm{Cl}: 1.15-2.16) ; \\
\text { Lower academic performance } \\
(\mathrm{OR}=1.69 ; 95 \% \mathrm{Cl}: 1.34-2.15) \\
\text { Internet risks behaviors: } \\
\text { Posting personal information photos } \\
\text { and using a webcam to chat with } \\
\text { strangers: } \\
\text { Cy-b (OR = 1.87; } 95 \% \mathrm{Cl}: 1.28-2.73) ; \\
\text { Cy-v (OR = 1.58; } 95 \% \mathrm{Cl}: 1.28-1.96) ; \\
\text { Cy-v/Cy-b (OR }=4.28 ; 95 \% \mathrm{Cl}: 3.00- \\
6.10)\end{array}$ & $\begin{array}{l}\text { Rosenberg Self-esteem Scale } \\
\text { (Rosenberg } 43 \text { ); } \\
\text { Depression CES-D (Radloff 44) }\end{array}$ & $\begin{array}{l}\text { Low self-esteem scores and high } \\
\text { depression scores *: } \\
\text { Cy-v: low self-esteem scores and } \\
\text { high depression scores; } \\
\text { Cy-b: high depression scores; } \\
\text { Cy-b/Cy-v: low self-esteem and } \\
\text { high depression scores }\end{array}$ \\
\hline
\end{tabular}

Cy-v: cybervictims; Cy-b: cyberbullies; Cy-b/Cy-v: cybervictims/cyberbullies; NI: not informed; R2: coefficient of multiple correlation.

* Odds ratio for mental health problems was not informed.

Adolescents who were victims of cyberbullying and traditional school bullying reported more depressive symptoms and higher scores on the suicidal ideation and suicidal behavior scale $(\mathrm{OR}=1.5)$, as well as more suicide attempts that demanded medical treatment $(\mathrm{OR}=2.1) 21$. Hinduja \& Patching's 21 study showed that the likelihood of attempting suicide was up to twice as high among victims and aggressors, as compared to those not involved in cyberbullying $(\mathrm{OR}=1.5$; $\mathrm{OR}=2.1$, respectively). In this review, all types of traditional bullying and cyberbullying were associated with increased suicidal thoughts among victims and offenders 18,20,21 (Table 2).

\section{Discussion}

Cyberbullying occurs at a lower prevalence than other traditional or school bullying. However, cyberbullying affects a significant portion of adolescents (10 to $20 \%$ ), being related to emotional stress and negative emotions such as anger, fear, stress depression 13,16,17,22. Cyberbullies and cybervictims reported more experiences with school bullying, suggesting continuity and overlapping between traditional bullying and cyberbullying 17,18. Considering the importance of virtual spaces for interaction and for the psychosocial development of adolescents, the results suggest that negative interactions in a virtual environment could mediate the broad range of disturbances associated with bullying and cyberbullying: academic and psychosocial problems, depression, low self-esteem, and externalized hostility, among others.

Studies included in this review identified several psychosocial risk factors and mental health problems associated with cyberbullying among victims, offenders, and those who are, at the same time, victims and offenders. Cyberbullying is associated with emotional stress 13,16,17, social anxiety 15, substance use 20, depressive symptoms $13,14,18,19$, suicidal ideation and suicide attempts 18,20,21.

Emotional responses of adolescents exposed to cyberbullying vary in intensity and quality. In a multicenter study, that assessed the emotional impact of different forms of traditional bullying and cyberbullying, $68.5 \%$ of adolescents expe- 
rienced some negative emotions such as anger, upset, worry, stress, fear and "depressive feelings", while $24.5 \%$ of adolescents did not care about the incidents 22 . The emotional impact is harmful to most victims, however adaptive resilience and a positive appraisal of a stressful situation or developmental strengths like self-esteem and self-efficacy could minimize the emotional damage to some victims. Further research should investigate which resilience factors could buffer the impact of negative experiences such as cyberbullying and bullying among adolescents 23 .

Anger was the most common reported emotion among adolescents in the study cited previously 22 . Situations like "posted things about me that I do not want anyone to know" harm the victims' reputation, publicly humiliate them or damage their friendships and social status in a stage of life in which the social group has an important role in shaping identity 1 . Anger has been considered a reaction to violations of autonomy and serves to facilitate a vigorous response to address the danger when an action has a negative impact on the "self" 24 . Therefore, in this context, angry reactions, could be considered a "healthy response" to cyberbullying.

Cybervictims may suffer verbal and visual anonymous attacks (photomontage) and anonymity can increase the feeling of fear and insecurity among the victims. When the perpetrator was an adult or an unknown person or group of persons, the victims also reported fear for their personal safety 9,15. Online aggressions can occur at any time, in the victim's own house, a safe environment. Most teens do not report the incident to adults, and the main reason reported by them has been that "they need to learn to deal with their own problems" (50\%), and also because they fear their parents would restrict their access to the Internet (31\%), leading to social isolation $14,25,26$.

Adolescents who reported experiences of cyberbullying, particularly those who suffered frequent attacks (two or more times a month), had more severe depressive symptoms when compared with adolescents exposed to other forms of bullying 18 . The feelings of helplessness and powerlessness to defend themselves from incidents of cyberbullying can increase the sense of fear and emotional distress, contributing to the emergence of depressive symptoms. The association between bullying, cyberbullying and depressive symptoms found in several studies 13,14,18,19 suggests that these phenomena occur in a bidirectional way (i.e., they can be either the cause or the consequence of each other). Cognitive impairment and depressive mood can change the perception of victims and the lack of social cues in online communication, such as volume and tone, can result in negative interpretations of ordinary situations, which can be perceived as threatening.

Cyberbulliyng may have even more harmful outcomes to adolescents' mental health, including substance abuse, increased suicidal ideation and suicide attempts 20,21. The Hinduja \& Patching 21 study showed that the likelihood of committing suicide attempts is up to twice as high among victims and aggressors, as compared to those not involved in cyberbullying. Having experienced cyberbullying alone does not induce suicide. Nevertheless, adolescents who have suffered cyberbullying may experience negative psychological states and abuse of alcohol and other drugs as a way to deal with negative feelings related to the aggression. This explanation is in agreement with the drug abuse etiology in adolescence. The use of psychoactive substances may also help adolescents to habituate to the physical pain and anxiety associated with automutilation. Using substances can also encourage adolescents with suicidal ideation, increasing auto-mutilation behaviors, lessening the inhibition and aggravating negative pre-existing moods $27,28,29$.

The adapted model showed in Figure 2 illustrates how drug use and violent behavior can mediate the relationship between cyberbullying, physical bulling and suicidal conduct in adolescents.

It is unlikely that experiences of cyberbullying alone lead to youth suicide. Rather, they may exacerbate an adolescent's instability and hopelessness at a time when they are already struggling with stressful life circumstances. Future research should identify the nature of this stress inducing experience. Studies regarding the differences found between adolescents facing cyberbullying, such as those who "do not care" and those "strongly impaired", may bring important contributions to strategies of prevention and intervention. Prevention strategies have to be implemented in order to avoid risk behaviors and aid adolescents to find effective ways of handling cyberbullying incidents and to avoid risk behaviors, such as exposing personal information, photos and webcam use with strangers, which can raise cyberbullying incidents and mental health damage $30,31,32$.

\section{Limitations}

Cyberbullying is a new concept in the literature and the methodology applied to investigate it is still a subject of discussion. A variety of terms 
are used including "online bullying”, "electronic bullying", "internet harassment" and "cyberbullying”. For this reason the search of articles was carried out using a comprehensive methodology. However, articles from two relevant databases such as PsycINFO and Social Science Citation Index were not considered for this review.

Most of the studies developed specific questions and evaluated the frequency of cyberbullying in different ways, depending on the researchers' objectives. Differences in definitions and, consequently, in the forms of measurement may impact on the estimates of prevalence of cyberbullying and of its impact on mental health.

Evaluating violent behaviors, which are not socially accepted may have influenced the adolescents answers and biased the cyberbulling prevalences and their association with mental health problems. Finally the causal direction of the association between cyberbullying and mental health problems cannot be determined by transversal studies included in this review.

\section{Resumen}

Se revisa la asociación entre el acoso cibernético y la salud mental de los adolescentes. Se realiza una revisión sistemática de dos bases de datos: PubMed y la Biblioteca Virtual en Salud (BVS). La prevalencia de ciberacoso varió de un 6,5\% a un $35,4 \%$. Los acosos cibernéticos tradicionales -pasados o actuales- se asociaron con las víctimas y los acosadores cibernéticos. El uso diario de tres o más horas de Internet, cámara web, mensajes de texto, la publicación de información personal y acosar a los demás se asociaron con el acoso cibernético. Cibervíctimas y acosadores cibernéticos tenían más problemas emocionales, psicosomáticos, dificultades sociales y no se sentían seguros y cuidados en la escuela. El ciberacoso se asoció con síntomas de moderados a graves de depresión, abuso de sustancias, ideación suicida e intentos de suicidio. Los profesionales de salud deben conocer la naturaleza violenta de las interacciones que se producen en el entorno virtual y sus peligros para la salud mental de los adolescentes.

Acoso; Adolescente; Salud Mental

\section{Conclusions}

Online communication has become a centerpiece in the life of adolescents, offering many opportunities for psychosocial development and construction of intimate relationships. However, in this context, violent interactions such as cyberbullying may occur.

Cyberbullying is associated with emotional stress, social anxiety, substance use, depressive symptoms, suicidal ideation and suicide attempts. Parents and educators ought to know the risks of on-line communication and need to promote dialogue about the topic, aiding adolescents to find effective ways to deal with such incidents. Health professionals must be aware of the occurrence of cyberbulling and its association with mental health problems.

\section{Contributors}

S. M. B. Bottino conceived the initial idea for the article, carried out the search, analyzed the results and wrote the article. C. M. C. Bottino supported in the article write-up, revision and answers to partners. C. G. C. Regina collaborated with the initial selection of articles, applied the eligibility criteria, and supported the revision and analysis of results. A. V. L. Correia contributed towards the results analysis and revision of the final text of the article. W. S. Ribeiro collaborated with the methodology, revision and application of eligibility criteria, and carried out the revision and correction of the final version of the article.

\section{Acknowledgments}

We wish to acknowledge the support of the Department of Psychiatry at the Federal University of São Paulo and Academic League for Violence Prevention and Intervention (LAPIV). 


\section{References}

1. Valkenburg PM, Peter J. Online communication among adolescents: an integrated model of its attraction, opportunities, and risks, J Adolesc Health 2011; 48:121-7.

2. Suzuki K, Asaga R, Sourander A, Hover CW, Mandel D. Cyberbullying and adolescent mental health. Int J Adolesc Med Health 2012; 24:27-35.

3. Monks C, Smith PK. Definitions of "bullying": age differences in understanding of the term, and the role of experience. Br J Dev Psychol 2006; 24: 801-21.

4. Due P, Holstein BE, Lynch J, Diderichsen F, Gabhain SN, Scheidt P, et al. Bullying and symptoms among school-aged children: international comparative cross sectional study in 28 countries. Eur J Public Health 2005; 15:128-32.

5. Nansel TR, Craig W, Overpeck MD, Saluja G, Ruan WJ; the Health Behavior in School-Age Children Bullying Analyses Working Group. Cross-national consistency in the relationship between bullying behavior and psychosocial adjustment. Arch Pediatr Adolesc Med 2004; 158:730-6.

6. Hawker DSJ, Boulton MJ. Twenty years' research on peer victimization and psychosocial maladjustment: a meta-analytic review of cross-sectional studies. J Child Psychol Psychiatry 2000; 41:441-55.

7. Smith PK, Mahdavi J, Carvalho M, Fisher S, Russell S, Tippett N. Cyberbullying: its nature and impact in secondary school pupils. J Child Psychol Psychiatry 2008; 49:376-85.

8. Tokunaga RS. Following you home from school: a critical review and synthesis of research on cyberbullying victimization. Comput Human Behav 2010; 26:277-87.

9. Nocentini A, Calmaestra J, Schultze-Krumbholz A, Scheithauer H, Ortega R, Menesini E. Cyberbullying: labels, behaviors and definition in three European countries. Australian Journal of Guidance and Counselling 2010; 20:129-42.

10. Dehue F, Bolman C, Vollink, T. Cyberbullying: youngsters' experiences and parental perception. Cyberpsychol Behav 2008; 11:217-23.

11. Moher D, Liberati A, Tetzlaff J; PRISMA Group. Reprint - preferred reporting items for systematic reviews and meta-analyses: the PRISMA statement. Phys Ther 2009; 89:873-80.

12. National Collaborating Centre for Methods and Tools. Quality assessment tool for quantitative studies method. Hamilton: McMaster University; 2008.

13. Ybarra ML. Linkages between depressive symptomatology and internet harassment among young regular internet users. Cyberpsychol Behav 2004; 7:247-57.

14. Chang FC, Lee CM, Chiu CH, Hsi WY, Huang TF, Pan YC. Relationships among cyberbullying, school bullying, and mental health in Taiwanese adolescents. J Sch Health 2013; 83:454-62.

15. Juvonen J, Gross EF. Extending the school grounds? Bullying experiences in cyberspace. J Sch Health 2008, 78:496-505.
16. Ybarra ML, Mitchell KJ, Wolak J, Finkelhor D. Examining characteristics and associated distress related to internet harassment: findings from the Second Youth Internet Safety Survey. Pediatrics 2006; 118:1169-77.

17. Sourander A, Brunstein Klomek A, Ikonen M, Lindroos J, Luntamo T, Koskelainen M, et al. Psychosocial risk factors associated with cyberbullying among adolescents: a population-based study. Arch Gen Psychiatry 2010; 67:720-8.

18. Schneider SK, O’Donnell L, Stueve A, Coulter RWS. Cyberbullying, school bullying, and psychological distress: a regional census of high school students. Am J Public Health 2012; 102:171-7.

19. Wang J, Nansel TR, Iannotti RJ. Cyber and traditional bullying: differential association with depression. J Adolesc Health 2011; 48:415-7.

20. Litwiller B, Brausch A. Cyber bullying and physical bullying in adolescent suicide: the role of violent behavior and substance use. J Youth Adolesc 2013; 42:675-84

21. Hinduja S, Patchin JW. Bullying, cyberbullying and suicide. Arch Suicide Res 2010; 14:206-21.

22. Ortega R, Elipe P, Mora-Merchan JA, Genta ML, Brighi A, Guarini A, et al. The emotional impact of bullying and cyberbullying on victims: a European Cross-National Study. Aggress Behav 2012; 38:342-56.

23. Hunter SC, Boyle JM, Warden D. Help seeking amongst child and adolescent victims of peeraggression and bullying: the influence of schoolstage, gender, victimisation, appraisal, and emotion. Br J Educ Psychol 2004; 74:375-90.

24. Hutcherson CA, Gross JJ. The moral emotions: a social-functionalist account of anger, disgust, and contempt. J Pers Soc Psychol 2011; 100:719-37.

25. Patchin WJ, Hinduja S. Bullies move beyond the schoolyard: a preliminary look at cyberbullying. Youth Violence Juv Justice 2006; 4:148-69.

26. Slonje R, Smith PK. Cyberbullying: another main type of bullying? Scand J Psychol 2008; 49:147-54.

27. Spirito A, Mehlenbeck R, Barnett N, Lewander W, Voss A. The relation of mood and behavior to alcohol use in adolescent suicide attempters. J Child Adolesc Subst Abuse 2003; 12:35-53.

28. Gould MS, King R, Greenwald S, Fisher P, SchwabStone M, Kramer R, et al. Psychopathology associated with suicidal ideation and attempts among children and adolescents. J Am Acad Child Adolesc Psychiatry 1998; 37:915-23.

29. Bauman S, Toomey RB, Walker JL. Associations among bullying, cyberbullying, and suicide in high school students. J Adolesc 2013; 36:341-50.

30. Pérez JC, Lecannelier FA. Evaluación de la efectividad del Programa Vínculos para la prevención e intervención del bullying en Santiago de Chile. Psicol Esc Educ 2013; 17:163-72.

31. Hui EKP, Tsang KM, Law BCM. Combating school bullying through developmental guidance for positive youth development and promoting harmonious school culture. ScientificWorldJournal 2011; 11:2266-77. 
32. Bostic JQ, Brunt CC. Cornered: an approach to school bullying and cyberbullying, and forensic implications. Child Adolesc Psychiatric Clin N Am 2011; 20:447-65.

33. Genta ML, Smith PK, Ortega R, Brighi A, Guarini A, Thompson F, et al. Comparative aspects of cyberbullying in Italy, England and Spain: findings from a DAPHNE Project. In: Li Q, Cross D, Smith PK, editors. Cyberbullying in the global playground: research from international perspectives. Chichester: John Wiley and Sons; 2012. p. 15-31.

34. American Psychiatric Association. Diagnostic and statistical manual of mental disorders, 4th Ed. Washington DC: American Psychiatric Association; 1999.

35. Achenbach TM. Manual for the youth self-report and profile. Burlington: Department of Psychiatry, University of Vermont; 1991.

36. La Greca AM, Lopez N. Social anxiety among adolescents: linkages with peer relations and friendships. J Abnorm Child Psychol 1998; 26:83-94.

37. American School Health Association. The National Adolescent Student Health Survey: a report on the health of America's youth. Oakland: Third Party Publishing; 1989.

38. Goodman R. The strengths and difficulties questionnaire: a research note. J Child Psychol Psychiatry 1997; 38:581-6.

39. Dahlberg LL, Toal SB, Swahn M, Behrens CB. Measuring violence-related attitudes, behaviors, and influences among youths: a compendium of assessment tools. 2nd Ed. Atlanta: Centers for Disease Control and Prevention; 2005.
40. Centers for Disease Control and Prevention. Youth risk behavior survey. http://www.cdc.gov/ HealthyYouth/yrbs/questionnaire_rationale.htm (accessed on 12/Apr/2010).

41. Massachusetts Department of Elementary and Secondary Education; Massachusetts Department of Public Health. Health and risk behaviors of Massachusetts youth, 2007: the report. Malden: Massachusetts Department of Elementary and Secondary Education/Massachusetts Department of Public Health; 2008.

42. Johnston LD, O’Malley PM, Bachman JG, Schulenberg JE. Monitoring the Future: national results on adolescent drug use. Overview of key findings, 2008. Bethesda: National Institute on Drug Abuse; 2009. (NIH Publication, 09-7401).

43. Rosenberg M. Society and the adolescent self-image. Princeton: Princeton University Press; 1965.

44. Radloff LS. The CES-D scale: a self-report depression scale for research in the general population. Appl Psychol Meas 1977; 1:385-40.

Submitted on 10/Mar/2014

Final version resubmitted on 15/Oct/2014

Approved on 17/Nov/2014 